\title{
AVALIAÇÃO ECONÔMICA DE PLANTAÇÕES DE EUCALIPTO SUBMETIDAS A DESBASTE ${ }^{1}$
}

\author{
Thelma Shirlen Soares², Márcio Lopes da Silva³, João Ricardo Vasconcelos Gama², Rosa Maria Miranda Armond \\ Carvalho $^{2}$ e Rodrigo Silva do Vale ${ }^{2}$
}

\begin{abstract}
RESUMO - O objetivo deste estudo foi determinar, através de métodos de avaliação econômica, a idade econômica de corte de plantações de eucalipto submetidas a desbaste. Para tanto, foram utilizados dados de um experimento de desbastes estabelecido na região nordeste da Bahia. Foram consideradas as variações de intensidades de desbaste $(0,20,40$ e $60 \%$ de retirada em área basal) para projeções realizadas em diferentes classes de local. O método de avaliação econômica utilizado foi o valor anual equivalente. $\mathrm{Na}$ análise de sensibilidade foram levadas em conta taxas de desconto de 6,9 e $12 \%$. Os resultados evidenciaram que a rotação econômica propicia maior lucratividade quando se realiza desbaste.
\end{abstract}

Palavras-chave: Idade ótima de corte, remoção de área basal e rotação econômica.

\section{ECONOMIC EVALUATION OF EUCALYPTUS STANDS SUBMITTED TO THINNING}

\begin{abstract}
This study aimed to determine, through economic evaluation methods, the effect of different thinning intensities on the economic rotation of eucalyptus stands. Data of a thinning experiment in northeastern Bahia' were used. Thinning intensity variations were considered (removal of 0, 20, 40 and 60\% in the basal area) for projections conducted in different sites. The Equivalent Annual Value was used as the decision variable. Discount rates of 6\%, 9\% and 12\% were considered in the sensibility analysis. The results showed that economic rotation provides a greater profitability with thinning.
\end{abstract}

Key words: Optimal age of harvest, removal of basal area, and economic rotation.

\section{INTRODUÇÃO}

Como forma de aproveitar áreas já desmatadas e diminuir a pressão exercida sobre as florestas nativas, bem como atender a mercados específicos, cada vez mais as florestas plantadas passam a desempenhar um importante papel como fonte alternativa de matéria-prima para a indústria florestal.

Segundo Silva et al. (1999), no Brasil os povoamentos de eucalipto têm sido mais utilizados como termorredutor para o setor siderúrgico e como fonte de matéria-prima para fabricação de celulose, pelo fato de sua madeira ser considerada de qualidade inferior. Atualmente a pesquisa vem rompendo este paradigma, pois já existem trabalhos que mostram a multiplicidade de produtos possíveis de serem obtidos a partir dos povoamentos de eucalipto, o que permite a empresa florestal direcionar suas atividades para a produção de madeira serrada, laminados, aglomerados, entre outros produtos.

A qualidade e a produtividade dos povoamentos florestais são os fatores mais importantes para a conquista de bons mercados e para obter um fluxo contínuo de matéria-prima. Para que isto seja conseguido é necessário

1 Recebido para publicação em 25.3.2002.

Aceito para publicação em 9.9.2003.

2 Estudante de Doutorado do Curso Ciência Florestal da Universidade Federal de Viçosa - UFV, 36570-000 Viçosa-MG;

${ }^{3}$ Professor do Departamento de Engenharia Florestal - UFV. 
executar tratos silviculturais, entre os quais o desbaste é um dos mais importantes, por proporcionar espaço para o desenvolvimento da copa e do sistema radicular e, conseqüentemente, melhor aproveitamento de luz, nutrientes e água disponíveis no sítio.

Entretanto, desbastes intensos podem levar ao aumento desproporcional do tamanho da copa e dos galhos, vindo a reduzir a qualidade da madeira, bem como, em geral, reduzem a produção volumétrica da floresta. Por outro lado, desbastes de intensidade adequada, aplicados no momento certo, permitem melhorar a qualidade da madeira, homogeneizar os sortimentos e aumentar a dimensão das árvores, sem levar a perdas significativas de volume (Schneider et al., 1998).

Para que se possa ter maior segurança quanto ao sucesso desses novos produtos, é importante determinar a idade econômica de corte da floresta, também denominada de rotação econômica, que é a idade de corte da floresta que maximiza o lucro. Assim, para calculá-la deve-se levar em consideração o valor do volume de madeira produzido em função do horizonte de planejamento.

Existem vários fatores que podem influenciar a idade econômica de corte, como: o horizonte de planejamento, os custos envolvidos na produção, o preço da madeira, a produtividade do local e a taxa de desconto utilizada. Todos estes fatores fazem parte de um conjunto de informações que o economista florestal deve dispor para avaliar economicamente qualquer projeto de investimento florestal (Lima Júnior et al., 1999).

Diante do exposto, este estudo teve como objetivo determinar, através de métodos de avaliação econômica, a idade econômica de corte de plantações de eucalipto submetidas a desbaste.

\section{MATERIAL E MÉTODOS}

\subsection{Fonte de Dados}

Os dados utilizados foram obtidos do estudo de Dias (2000), realizado na região nordeste da Bahia, onde foi instalado um experimento de desbaste estabelecido em povoamentos do híbrido Eucalyptus urophylla vs Eucalyptus grandis, nos espaçamentos $3,5 \times 2,6 \mathrm{~m}$ e $2,9 \times 3,0 \mathrm{~m}$.

A equação utilizada para gerar a tabela de produção de densidade variável, nas diferentes classes de local, foi obtida a partir do modelo de Clutter (1963), ajustado por Dias (2000). A relação funcional da equação empregada é

$$
\begin{aligned}
& \ln V_{2}=2,441896-24,23312 \frac{1}{I_{2}}+0,032370 \mathrm{~S}+0,726114 \ln B_{2} \\
& \ln B_{2}=\ln B_{1}\left(\frac{I_{1}}{I_{2}}\right)+3,286565\left(1-\frac{I_{1}}{I_{2}}\right)
\end{aligned}
$$

em que

$V_{2}=$ volume futuro $\left(\mathrm{m}^{3} / \mathrm{ha}\right) ;$

$I_{1}=$ idade atual (meses);

$I_{2}=$ idade futura (meses);

$B_{1}=$ área basal atual $\left(\mathrm{m}^{2} / \mathrm{ha}\right)$;

$B_{2}=$ área basal futura $\left(\mathrm{m}^{2} / \mathrm{ha}\right)$;

$S=$ índice de local (m); e

$\ln =$ logaritmo neperiano.

Uma vez devidamente avaliado, o modelo foi utilizado por Dias (2000) para elaborar a tabela de produção volumétrica para projeções realizadas nas diferentes classes de local, conforme apresentado no Quadro 1.

\subsection{Simulação de Desbastes e Análise Econômica}

Para análise da produção volumétrica, foram estabelecidas simulações de desbastes na tabela de produção, com variações de intensidades de desbaste $(0,20,40$ e $60 \%$ de retirada em área basal), para projeções realizadas nas diferentes classes de local.

Cada intensidade de desbaste foi considerada, para fins de análise, como um projeto. Para comparação dos projetos utilizou-se como método de avaliação econômica o valor anual equivalente (VAE).

O VAE é a parcela periódica e constante necessária ao pagamento de uma quantia igual ao VPL da opção de investimento em análise, ao longo de seu horizonte de planejamento. Este critério consiste em determinar a renda, ou benefício, se for positivo, ou o custo, se for negativo, equivalente por período de vida útil do projeto. Seu cálculo é dado por:

$V A E=\frac{\left.V P L\left[(1+i)^{t}-1\right] 1+i\right)^{n t}}{(1+i)^{n t}-1}$ 
Quadro 1 - Tabela de produção de densidade variável Table 1 - Variable density production

\begin{tabular}{|r|c|c|c|}
\hline $\begin{array}{c}\text { Idade } \\
\text { (meses) }\end{array}$ & $\begin{array}{c}\text { Área Basal } \\
\left(\mathrm{m}^{2} / \mathrm{ha}\right)\end{array}$ & $\begin{array}{c}\text { Volume } \\
\left(\mathrm{m}^{3} / \mathrm{ha}\right)\end{array}$ & $\begin{array}{c}\text { IMM* } \\
\left(\mathrm{m}^{3} / \mathrm{ha} / \mathrm{ano}\right)\end{array}$ \\
\hline \multicolumn{4}{|c|}{ Classe de Local I $(\mathrm{S}=25)$} \\
\hline 27 & 9,00 & 51,89 & 1,922 \\
36 & 11,82 & 79,14 & 2,198 \\
60 & 16,38 & 131,33 & 2,189 \\
84 & 18,85 & 163,18 & 1,943 \\
108 & 20,37 & 184,09 & 1,705 \\
132 & 21,41 & 198,78 & 1,506 \\
156 & 22,15 & 209,63 & 1,344 \\
\hline \multicolumn{4}{|c|}{ Classe de Local II $(\mathrm{S}=21)$} \\
\hline 27 & 8,50 & 43,73 & 1,620 \\
36 & 11,32 & 67,40 & 1,872 \\
60 & 15,97 & 113,25 & 1,887 \\
84 & 18,51 & 141,46 & 1,684 \\
108 & 20,08 & 160,07 & 1,482 \\
132 & 21,16 & 173,16 & 1,312 \\
156 & 21,94 & 182,85 & 1,172 \\
\hline \multicolumn{4}{|c|}{ Classe de Local III (S =17) } \\
\hline 27 & 7,50 & 35,08 & 1,299 \\
36 & 10,31 & 55,31 & 1,536 \\
60 & 15,09 & 95,51 & 1,592 \\
84 & 17,78 & 120,70 & 1,437 \\
108 & 19,47 & 137,47 & 1,273 \\
132 & 20,62 & 149,33 & 1,131 \\
156 & 21,47 & 158,14 & 1,014 \\
\hline
\end{tabular}

* IMM = incremento médio mensal.

em que $V P L=$ valor presente líquido $=$

$$
\sum_{j=1}^{n} \frac{R_{j}}{(1+i)^{j}}-\sum_{j=1}^{n} \frac{C_{j}}{(1+i)^{j}}
$$

$R_{\mathrm{j}}=$ receita líquida ao final do ano ou do período de tempo considerado;

$C_{j}=$ custos ao final do ano ou do período de tempo considerado;

$i=$ taxa de desconto;

$n=$ duração do projeto em meses; e

$t=$ número de períodos de capitalização.

Um projeto é considerado viável economicamente se o $V A E$ for positivo, o que indica que os benefícios periódicos são maiores do que os custos periódicos. Por outro lado, ao comparar dois ou mais projetos, será mais viável economicamente aquele que apresentar o maior VAE. Este método é útil para comparar projetos com horizontes de planejamento de diferentes durações, caso em que o VPL não é apropriado.

$\mathrm{Na}$ análise econômica, foram consideradas uma taxa de desconto de $10 \%$ a.a. e a ocorrência de desbaste aos 60 meses.

\subsection{Custos e Receitas Envolvidos na Produção Florestal}

Para determinar a rotação econômica é necessário conhecer todos os custos e receitas decorrentes do processo de formação e condução do povoamento, bem como a sua distribuição ao longo do tempo (Silva et al., 1995). No Quadro 2 estão os itens de custo médio para as diversas operações envolvidas nos povoamentos em estudo.

Quadro 2 - Itens de custo dos projetos em análise Table 2 - Cost components of the projects analyzed

\begin{tabular}{|l|c|c|}
\hline \multicolumn{1}{|c|}{ Atividade } & Ano de Ocorrência & Custo (U\$\$/ha) \\
\hline Implantação & 0 & 400,00 \\
Capina & 1 & 50,00 \\
Capina & 2 & 20,00 \\
Manutenção & Anualmente & 10,00 \\
Colheita desbaste & 5 & $10,00^{*}$ \\
Colheita final & $\mathrm{n}$ & 7,00 \\
Valor da terra & - & 350,00 \\
Administração & - & $10 \%$ do custo total \\
\hline
\end{tabular}

* Valores em US $\$ \mathrm{~m}^{3}$, sendo US $\$ 1.00=\mathrm{R} \$ 2,50$.

As receitas consideradas na realização das análises foram aquelas provenientes da venda da madeira no momento do desbaste e no corte final. Elas ocorreram no ano em que foram efetuados o desbaste e a colheita.

Neste estudo, considerou-se que o preço de venda da madeira varia em função da idade do povoamento. Assim, para obter a taxa de variação do preço ao longo dos anos empregou-se a fórmula de juros compostos, através da expressão:

$$
c=\sqrt[n]{\frac{P_{n}}{P_{0}}}-1
$$

R. Árvore, Viçosa-MG, v.27, n.4, p.481-486, 2003 
em que

$c=$ taxa de variação do preço;

$P_{n}=$ preço final (US\$);

$P_{0}=$ preço inicial (US\$); e

$n=$ número de períodos de capitalização.

Para madeira advinda de povoamento desbastado, foram estipulados preços de US\$8.00 e US\$25.00/ $\mathrm{m}^{3}$ nas idades de 5 e 25 anos, respectivamente, obtendo-se uma taxa de variação do preço de 5,86\% a.a. Já para madeira advinda de povoamento não-desbastado, foi considerado o preço de US $\$ 8.00 / \mathrm{m}^{3}$, independentemente da idade final de corte.

\subsection{Análise de Sensibilidade}

Realizou-se uma análise de sensibilidade do VAE às variações da época e intensidade de desbaste e nas taxas de desconto, para estabelecer a influência destes fatores na viabilidade econômica dos projetos. Para efeito de análise, utilizou-se a classe de local II $(\mathrm{S}=21)$.

No caso da época de desbaste, considerou-se a sua ocorrência nas idades de 48, 60 e 72 meses, nas intensidades de 20, 40 e $60 \%$ de desbaste. Com relação às taxas de desconto, considerou-se a aplicação de taxas reais de desconto de 6,9 e $12 \%$ ao ano no cálculo do VAE. Essas taxas foram escolhidas por abrangerem os níveis mais usados pelo setor florestal (Oliveira et al., 1998).

\section{RESULTADOS E DISCUSSÃO}

\subsection{Análise Econômica}

A partir da avaliação econômica, considerando a não-realização de desbastes, verificou-se que a idade final de corte ocorreu aos 7, 8 e 10 anos para as classes de local I, II e III, respectivamente. Porém, considerando a ocorrência de desbaste aos 60 meses, verificou-se que a idade ótima de corte foi de 12 anos para remoção de 20 e $40 \%$ da área basal e 13 anos para $60 \%$ de remoção, na classe de local I. Para a classe de local II, o corte final ocorreu aos 13 anos para as intensidades de desbaste 20 e $40 \%$ e aos 14 anos para intensidade de $60 \%$. Já na classe de local III, a idade de corte final ocorreu aos 15 anos para todas as intensidades de remoção da área basal.

Os resultados do Quadro 3 mostram receita líquida positiva para todos os projetos que envolvem desbaste, independentemente da intensidade de desbaste e das características do povoamento. Porém, observa-se que quanto melhor a capacidade produtiva do local maior a lucratividade do projeto. No entanto, ocorrem decréscimos da receita líquida na proporção do aumento da intensidade de desbaste. Verifica-se, portanto, que do ponto de vista econômico é recomendável a realização de desbastes.

\subsection{Análise de Sensibilidade}

Analisando o efeito da taxa de desconto e do desbaste sobre a viabilidade econômica (Quadro 4), observase que, independentemente do porcentual de remoção da área basal considerado, a taxa de $6 \%$ a.a. é a que garante maiores lucros. Tal resultado já era esperado, uma vez que à medida que a taxa de desconto aumenta o risco do empreendimento também é maior e o VAE de todos os tratamentos cresce em termos negativos, ou seja, o prejuízo aumenta. As altas taxas de desconto tendem a inviabilizar investimentos a longo prazo, como é o caso de investimentos no setor florestal.

Oliveira et al. (1998) relataram que devido ao longo tempo de conversão em invetsimentos florestais a viabilidade de qualquer projeto apresenta considerável

Quadro 3 - Valor presente líquido (VPL) considerando taxa de desconto de $10 \%$ a.a. em diferentes classes de local

Table 3 - Net present value (VPL) regarding rate of discount of $10 \%$ a.a. in different sites

\begin{tabular}{|c|c|c|}
\hline $\begin{array}{c}\text { \% de Redução da } \\
\text { Área Basal }\end{array}$ & VAE* & $\mathrm{IOC}^{* *}$ \\
\hline \multicolumn{3}{|c|}{ Classe de local I $(\mathrm{S}=25)$} \\
\hline 0 & 81,85 & 7 \\
\hline 20 & 271,97 & 12 \\
\hline 40 & 266,27 & 12 \\
\hline 60 & 245,25 & 13 \\
\hline \multicolumn{3}{|c|}{ Classe de local II $(\mathrm{S}=21)$} \\
\hline 0 & 80,28 & 8 \\
\hline 20 & 208,88 & 13 \\
\hline 40 & 204,23 & 13 \\
\hline 60 & 187,40 & 14 \\
\hline \multicolumn{3}{|c|}{ Classe de local III $(S=17)$} \\
\hline 0 & 74,98 & 10 \\
\hline 20 & 145,63 & 15 \\
\hline 40 & 142,05 & 15 \\
\hline 60 & 138,11 & 15 \\
\hline
\end{tabular}

* Valores em US\$/ha e ** em anos. 
sensibilidade às variações na taxa real de desconto usada. Assim, os autores sugerem o uso de taxas inferiores àquelas usadas por outros setores da economia.

Quadro 4 - Valor atual equivalente (VAE) considerando mudanças nas taxas de desconto e idade de desbaste

Table 4 - Equivalent Current Value (VAE) regarding changes in the discount rates and age of thinning

\begin{tabular}{|c|c|c|c|c|}
\hline $\begin{array}{c}\text { Remoção da } \\
\text { Área Basal } \\
(\%)\end{array}$ & $\begin{array}{c}\text { Época de } \\
\text { Desbaste } \\
\text { (meses) }\end{array}$ & $\begin{array}{c}\text { Taxa de } \\
\text { Desconto } \\
(\%)\end{array}$ & $\begin{array}{c}\text { VAE } \\
\text { (US\$/ha) }\end{array}$ & $\begin{array}{l}\text { IOC } \\
\text { (anos) }\end{array}$ \\
\hline \multirow{9}{*}{20} & \multirow{3}{*}{48} & 6 & 340.33 & 13 \\
\hline & & 9 & 240.09 & 13 \\
\hline & & 12 & 151.81 & 13 \\
\hline & \multirow{3}{*}{60} & 6 & 333.71 & 14 \\
\hline & & 9 & 228.58 & 14 \\
\hline & & 12 & 137.24 & 14 \\
\hline & \multirow{3}{*}{72} & 6 & 327.82 & 15 \\
\hline & & 9 & 217.91 & 15 \\
\hline & & 12 & 123.84 & 15 \\
\hline \multirow{9}{*}{40} & \multirow{3}{*}{48} & 6 & 336.47 & 13 \\
\hline & & 9 & 236.05 & 13 \\
\hline & & 12 & 147.62 & 13 \\
\hline & \multirow{3}{*}{60} & 6 & 329.45 & 14 \\
\hline & & 9 & 224.18 & 14 \\
\hline & & 12 & 132.79 & 14 \\
\hline & \multirow{3}{*}{72} & 6 & 323.39 & 15 \\
\hline & & 9 & 213.40 & 15 \\
\hline & & 12 & 119.29 & 15 \\
\hline \multirow{9}{*}{60} & \multirow{3}{*}{48} & 6 & 326.62 & 14 \\
\hline & & 9 & 221.03 & 14 \\
\hline & & 12 & 129.33 & 14 \\
\hline & \multirow{3}{*}{60} & 6 & 319.69 & 15 \\
\hline & & 9 & 209.38 & 15 \\
\hline & & 12 & 114.98 & 15 \\
\hline & \multirow{3}{*}{72} & 6 & 313.90 & 16 \\
\hline & & 9 & 199.00 & 16 \\
\hline & & 12 & 102.17 & 16 \\
\hline
\end{tabular}

De acordo com os valores obtidos para o VAE, nas diferentes taxas de desconto e épocas de desbaste, verifica-se que ele indica maior viabilidade econômica para os projetos que realizam desbaste mais cedo, sendo o desbaste aos 48 meses o que gera maior rentabilidade.

Observa-se, também, que a opção que propicia maior retorno econômico é efetuar o desbaste na intensidade de $20 \%$ de remoção da área basal, aos 48 meses. A Figura 1 ilustra, de forma sintética, o comportamento das variáveis analisadas para o povoamento em estudo.

$$
\begin{aligned}
& \uparrow \% \text { desbaste } \Rightarrow \downarrow \text { lucratividade } \\
& \uparrow \text { capacidade produtiva } \Rightarrow \uparrow \text { lucratividade } \\
& \uparrow \text { taxa de desconto } \Rightarrow \downarrow \text { lucratividade }
\end{aligned}
$$

Figura 1 - Influência das variáveis estudadas sobre a lucratividade.

Figure $\mathbf{1}$ - Influence of the variables on profitability.

\section{CONCLUSÃO}

As principais conclusões deste estudo são:

- a opção mais lucrativa é remover $20 \%$ da área basal na idade de 60 meses;

- a rotação econômica, quando o desbaste foi efetuado aos 60 meses, ocorreu aos 12, 13 e 14 anos para as intensidades de desbaste 20 e $40 \%$, respectivamente;

- a rotação econômica, quando o desbaste foi efetuado aos 60 meses, ocorreu aos 13, 14 e 15 anos para a intensidades de desbaste $60 \%$; e

- a rotação econômica propicia menor lucratividade quando não são realizados desbastes.

\section{REFERÊNCIAS BIBLIOGRÁFICAS}

CLUTTER, J. L. Compatible growth and yield models for loblolly pine. Forest Science, v. 9, n. 3, p. 354-371, 1963.

DIAS, A. N. Modelagem e avaliação econômica de plantações de eucalipto submetidas a desbastes. 2000. 70 f. Dissertação (Mestrado em Ciência Florestal) Universidade Federal de Viçosa, Viçosa, 2000.

LIMA Jr., V. B.; REZENDE, J. L. P.; SILVA, M. L. Os estágios de produção e a idade ótima de corte: diferença entre a teoria da produção instantânea e a preferência temporal na produção florestal. Revista Árvore, v. 23, n. 4 , p. 393-401, 1999.

SCHNEIDER, P. R. et al. Produção de Eucalyptus grandis Hill ex Maiden em diferentes intensidades de desbaste.

Ciência Florestal, v. 8, n. 1, p. 129-140, 1998.

SILVA, M. L.; FONTES, A. A.; LEITE, H. G. Rotação econômica em plantações de eucalipto não-desbastadas e destinadas a multiprodutos. Revista Árvore, v. 23, n. 4, p. 403-412, 1999.

R. Árvore, Viçosa-MG, v.27, n.4, p.481-486, 2003 
SILVA, M. L.; MACHADO, C. C.; LADEIRA, H. P. Influência do custo de corte, do diâmetro da árvore e do volume por hectare na rotação econômica de povoamentos de eucalipto. Revista Árvore, v. 19, n. 4, p. 501-516, 1995.
OLIVEIRA, A. D. et al. Avaliação econômica da vegetação de cerrado submetida a diferentes regimes de manejo e de povoamentos de eucalipto plantado em monocultivo.

Revista Cerne, v. 4, n. 1, p. 34-56, 1998. 